\title{
Pan drug-resistant Acinetobacter baumannii causing nosocomial infections among burnt children
}

\author{
Behnam Sobouti ${ }^{1}$, Maryam Mirshekar ${ }^{2,3}$, Shahrzad Fallah ${ }^{4}$, Aram Tabaei ${ }^{1}$, Jalil Fallah Mehrabadi ${ }^{5}$, Atieh Darbandi*2,3(D) \\ Received: 4 Oct 2019 \\ Published: 23 Mar 2020
}

\begin{abstract}
Background: Nosocomial infection caused by Acinetobacter baumannii has emerged as a world-wide serious problem in the emergence of multidrug-resistant (MDR). Infections caused by antibiotic-resistant strains of $A$. baumannii cannot be completely eliminated among the infected patients. This study aimed to monitor antibiotic resistance among $A$. baumannii strains isolated from burnt children.

Methods: After performing biochemical identification tests on 115 isolates, 62 were detected as A. baumannii. Minimum inhibitory concentration (MIC) was used to test susceptibility to colistin, and disk agar diffusion was used for the susceptibility of the isolates to the antibiotics Ciprofloxacin, Amikacin, Gentamicin, Cefepime, Meropenem, Imipenem, Ceftazidime, Levofloxacin and Piperacillin/Tazobactam. Bacterial species were isolated and identified as multidrug-resistant (MDR), extensively drug-resistant (XDR) and pan drug-resistant (PDR), based on the susceptibility patterns to elected antibiotics, deputing different classes of antimicrobial.

Results: The antibiotic susceptibility pattern out of a total of 62 bacterial strains used in this study. Thirty-six (58\%) strains were categorized as MDR, $17(27.5 \%)$ as XDR, and nine (14.5\%) as PDR.

Conclusion: To reduce the threat of antimicrobial resistance, MDR, XDR and PDR A. baumannii strains must be evaluated by all clinical microbiology laboratories.
\end{abstract}

Keywords: PDR, Acinetobacter baumannii, Burnt Children

Conflicts of Interest: None declared

Funding: Iran University of Medical Science

*This work has been published under CC BY-NC-SA 1.0 license.

Copyright $\subseteq$ Iran University of Medical Sciences

Cite this article as: Sobouti B, Mirshekar M, Fallah Sh, Tabaei A, Fallah Mehrabadi J, Darbandi A. Pan drug-resistant Acinetobacter baumannii causing nosocomial infections among burnt children y. Med J Islam Repub Iran. 2020 (23 Mar);34:24. https://doi.org/10.47176/mjiri.34.24

\section{Introduction}

Acinetobacter baumannii induced nosocomial infections, caught during hospitalization, have emerged as a serious worldwide problem, especially among immune-compromised, burnt and ICU (intensive care unit) patients (1). Multidrug resistance among the hospital strains of this species has made it cumbersome, with immediate risk to the

Corresponding author: Dr Atieh Darbandi, darbandi.a@iums.ac.ir

- Burn Research Center, Iran University of Medical Sciences, Tehran, Iran

2. Student Research Committee, Iran University of Medical Sciences, Tehran, Iran

3. Department of Microbiology, School of Medicine, Iran University of Medical Sciences

Tehran, Iran

4. Shahid Beheshti University of Medical Sciences, Tehran, Iran

5. The Lister Laboratory of Microbiology, Tehran, Iran public health, to treat $A$. baumannii infections such as bacteremia, meningitis, pneumonia, and urinary tract infections (2).

MDR has been determined by European Center for Disease Prevention and Control (ECDC) and Center for Disease Control \& Prevention (CDC) as the accumulated non-

\section{$\uparrow$ What is "already known" in this topic:}

Acinetobacter baumannii infections in burn patients may lead to a lag in wound healing, graft losses, and development of septicemia. Furthermore, prevalence caused by multidrugresistant $A$. baumannii (MDRAB) is difficult to control and have fundamental morbidity and mortality, especially in the vulnerable hosts.

\section{$\rightarrow$ What this article adds:}

The current data indicated a high rate of resistance among $A$. baumannii isolates from burnt children. Since therapeutic options are limited for multidrug-resistant $A$. baumannii infections, finding new strategies for preventing healthcareassociated transmission of multidrug-resistant $A$. baumannii infections is urgent. 
susceptibility to at minimum one factor in three or more antimicrobial classes (3). XDR has been specified as nonsusceptibility to at minimum one factor in every but two or fewer antimicrobial classes (i.e., bacterial isolates subsist susceptible to only one or two antimicrobial classes). PDR has been specified as non-susceptibility to all factors in all antimicrobial classes (4). According to a previous study regarding the high prevalence of MDR, XDR and PDR strains of $A$. baumannii among Iranian patients (5), the current study was performed in order to monitor any increases in resistance among these isolates.

\section{Methods}

\section{Clinical Sampling and Bacterial Identification}

This short-term cross-sectional study was performed in Shahid Motahari Burns Hospital, Tehran, Iran from $15^{\text {th }}$ April to $15^{\text {th }}$ July 2016 . Totally, 115 isolates were studied from burn wound infections of inpatients involved in burning with different burn levels and identified by conventional methods (Fig. 1). The tissue culture was performed based on previously confirmed protocol $(5,6)$.

Briefly, biopsy samples were collected by $3 \mathrm{~mm}$ punch tissue with a weight between 0.02 and 0.05 gram. In order to prevent tissue samples desiccation during transportation, the biopsy samples were then placed on a non-bacteriostatic moistened sterile gauze pad within a sterile container. The biopsy samples were cut into small pieces and homogenized by a homogenizer (BioMaster-Stomacher, Sewerd, England). The suspension was serially diluted and $0.1 \mathrm{cc}$ sample was inoculated on 5\% defibrinated sheep blood Columbia agar, Eosin Methylene Blue agar and Chocolate agar, and incubated at $37^{\circ} \mathrm{C}$ for 24 hours. Standard microbiological tests in the laboratory were used to determine the isolates including Oxidase (negative), TSI (non-fermentative), Lysine decarboxylase (negative), lysine decarboxylase (positive), arginine hydrolase (positive), growth in 45 ${ }^{\circ} \mathrm{C}$, Gelatin hydrolysis (positive), hydrolysis of esculin agar, reduction of nitrate, production of acid from glucose in OF media, hemolysis reaction on blood agar. Bacterial strains were preserved in Trypticase Soy Broth (TSB) with $20 \%$ Glycerol $(7,8)$.

\section{Antibiotic Susceptibility Tests}

The disk diffusion method was used in order to detect antimicrobial susceptibility in clinical isolates of $A$. baumannii conforming to the Clinical and Laboratory Standards Institute (CLSI 2018) instructions (9, 10). The following antibiotics disks from MAST Categories Ltd., Merseyside, UK, were used: Ciprofloxacin (CIP, $5 \mu \mathrm{g}$ ), Amikacin (AK, $30 \mu \mathrm{g})$, Gentamicin (GM, $10 \mu \mathrm{g})$, Cefepime (CPM, $30 \mu \mathrm{g}$ ), Meropenem (MEM, $10 \mu \mathrm{g}$ ), Imipenem (IMI, 10 $\mu \mathrm{g})$, Ceftazidime (CAZ, $30 \mu \mathrm{g}$ ), Levofloxacin (LEV, $1 \mu \mathrm{g}$ ) and Piperacillin/Ttazobactam (PTZ, $10 \mu \mathrm{g}$ ). Then, the determined A. baumannii isolates were tested for Colistin susceptibility by minimum inhibitory concentrations (MIC). Pseudomonas aeruginosa ATCC27853 and Escherichia coli ATCC 25922 were used as a control for the susceptibility tests.

First, A. baumannii strains were cultured on TSB and incubated at $37^{\circ} \mathrm{C}$ for 24 hours. Subsequently, the turbidity of $0.5 \mathrm{McF}$ arland standards was used as a standard method and the bacterial suspension was adjusted with it. The sterile swabs were plated on Mueller-Hinton agar ( $\mathrm{MH}$ agar) and antibiotic disks were placed on the plate and incubated at $37^{\circ} \mathrm{C}$ for 24 hours.

MDR and XDR in A. baumannii isolates were defined according to new standardized international document (11), by the results of antimicrobial susceptibility of $P$. aeruginosa to all antimicrobial agents listed in Table 1.

Antimicrobial susceptibility of colistin against $\mathrm{A}$. baumannii isolates was investigated by the minimum inhibitory concentration (MIC) method. Colistin sulfate (SigmaAldrich, St. Louis, MO, USA) was tested over a range of dilutions $(64-0.5 \mu \mathrm{g} / \mathrm{ml})$. For this purpose, $100 \mu \mathrm{l}$ colistin sulfate (diluted in water) $128 \mathrm{mg} / \mathrm{ml}$ were added to 96 -well $\mathrm{U}$ bottom microplates. In the following $10 \mu \mathrm{l}$ of bacterial suspension containing $1.5 \times 10^{5}, \mathrm{CFU} / \mathrm{ml}$ of cells was added to wells with serially different concentrations of colistin. In the end, the microplate was incubated in ambient air at $37^{\circ} \mathrm{C}$ for $24 \mathrm{~h}$. The breakpoints of The Clinical and Laboratory Standards Institute (CLSI) were applied as references (12)

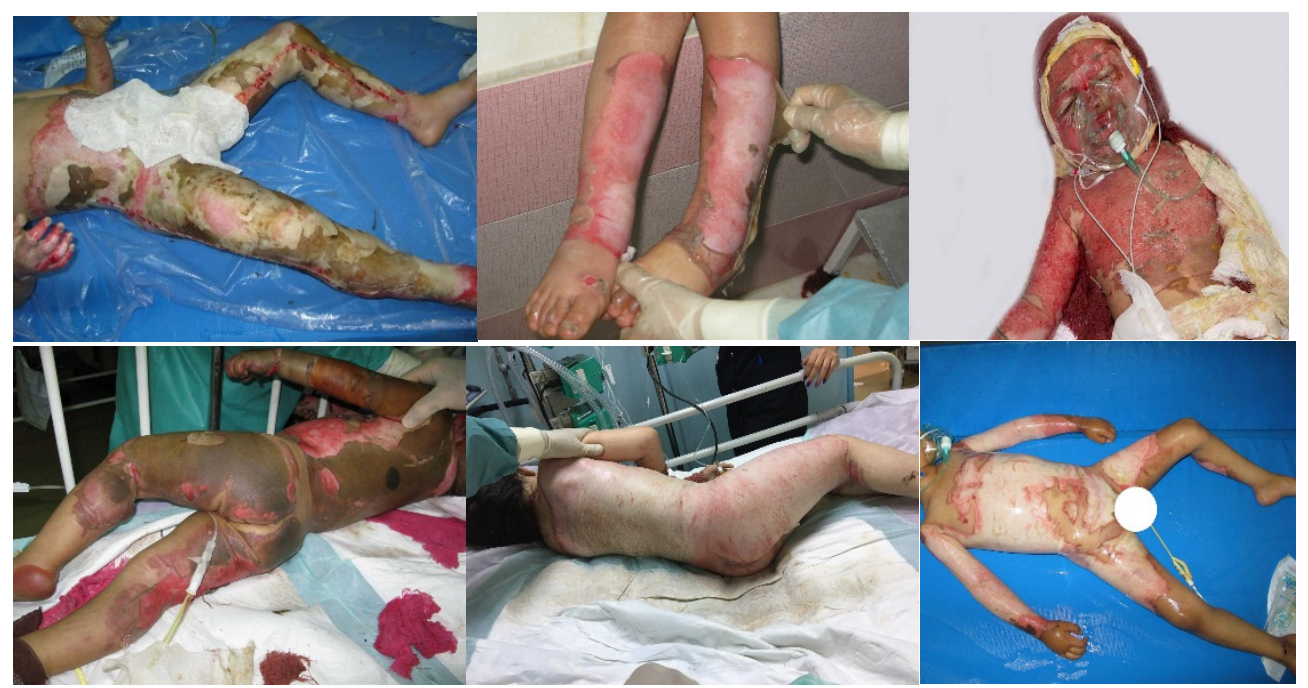

Fig. 1. A total of 115 isolates were studied during current survey in 2016 (The Medical Ethic Committee permitted us for releasing pictures) 


\begin{tabular}{|c|c|c|c|}
\hline Antimicrobial categories & Multi-drug resistant (MDR) & Extensively-drug resistant(XDR) & Pan--drug resistant(PDR) \\
\hline Definitions & $\begin{array}{l}\text { Resistance to at least } \\
\text { Three class of drug: } \\
\text { 1) Cephalosporins } \\
\text { 2) Fluroquinolons } \\
\text { 3) Aminoglycoside }\end{array}$ & $\begin{array}{c}\text { MDR } \\
\text { Acinetobacter } \\
+ \\
\text { Resistance to carbapenems }\end{array}$ & $\begin{array}{c}\text { XDR Acinetobacter } \\
+ \\
\text { Resistance to Polymyxins }\end{array}$ \\
\hline $\begin{array}{l}\text { Therapeutic } \\
\text { Options }\end{array}$ & $\begin{array}{l}\text { Carbapenems } \\
\text { Polymyxins }\end{array}$ & $\begin{array}{l}\text { Polymyxins } \\
\text { Tigecycline }\end{array}$ & Combinations \\
\hline
\end{tabular}

\section{Results}

Sixty-two (54\%) of bacterial isolates were distinguished as A. baumannii by means of standard microbiological tests in the laboratory. The mean age was $4.8 \pm 3.5$ years, including $34(55 \%)$ males and 28 (45\%) females. Two patients involved with PDR strains died (one male and one female).

According to antimicrobial susceptibility test, all isolates were resistant to ceftazidime $(n=62,100 \%)$, and then after ceftazidime, the most antibiotic resistance was related to ciprofloxacin $(n=50,81 \%)$. Least antibiotic resistance was related to levofloxacin $(n=12,19 \%)$. The rates of other antibiotic resistances are shown in Chart 1.

Moreover, the rate of antibiotic resistance to colistin was determined using MIC and elucidated in Table 2.

Consistent with definition of MDR, XDR and PDR strains (7), in this study, $36(58 \%)$ out of 62 isolates were recognized as MDR, 17 isolates $(27.5 \%)$ as XDR and nine $(14.5 \%)$ isolates were as PDR among 62 clinical isolates of $A$. baumannii isolates from patients in Tehran, Iran.

\section{Discussion}

The presence of highly resistant bacteria, including MDR, XDR and PDR in hospitals, especially in intensive care units (ICU) and burn centers is a major threat to the treatment of patients worldwide. These bacteria are able to cause nosocomial infections and continue to impose serious challenges to clinicians' empirical and therapeutic decisions to treat burnt patients $(13,14)$.
Outbreaks of MDR, XDR and PDR strains of $A$. baumannii are currently reported worldwide. Custovic et al. treated 855 patients in Bosnia and isolated 54 A. baumannii strains from 105 nosocomial infections. With an incidence of $51.4 \%(54 / 105)$, it was the predominant cause of infection among the patients. Most of these strains were resistant to most antibiotics except tobramycin $(87 \% ; 47 / 54)$ and colistin $(100 \% ; 54 / 54)(2)$. Some of the antibiotic resistance percentages in Custovic's study were similar to those in our study. The resistance rates of $A$. baumannii strains respectively found in Custovic's and our study included piperacillin/tazobactam 98.1\% (65\%), ciprofloxacin $96.2 \%$ (80\%), ceftazidime 94.4\% (100\%), cefepime 92.6\% (50\%), amikacin $92 \%(60 \%)$, meropenem $88.9 \%(50 \%)$ and gentamicin $60.4 \%(70 \%)$. The percentages in brackets are related to our study.

Hojabri et al. collected $71 \mathrm{~A}$. baumannii strains and analyzed them by reiterative extragenic palindromic PCR (repPCR), multi-locus sequence-based typing and an updated sequence group multiplex PCR. Sixty-seven (94.4\%) out of the 71 isolates were resistant to at least three antibiotic classes and were considered as MDR. Sixty (84.5\%) isolates were resistant together amikacin and carbapenems. About $65(91.5 \%)$ were determined resistant to gentamicin and also $67(94.4 \%)$ were resistant to both cefepime and ceftazidime (8). In contrast to our study which found PDR and XDR as well as MDR A. baumannii isolates, Hojabri only found MDR strains among the isolates. Another report

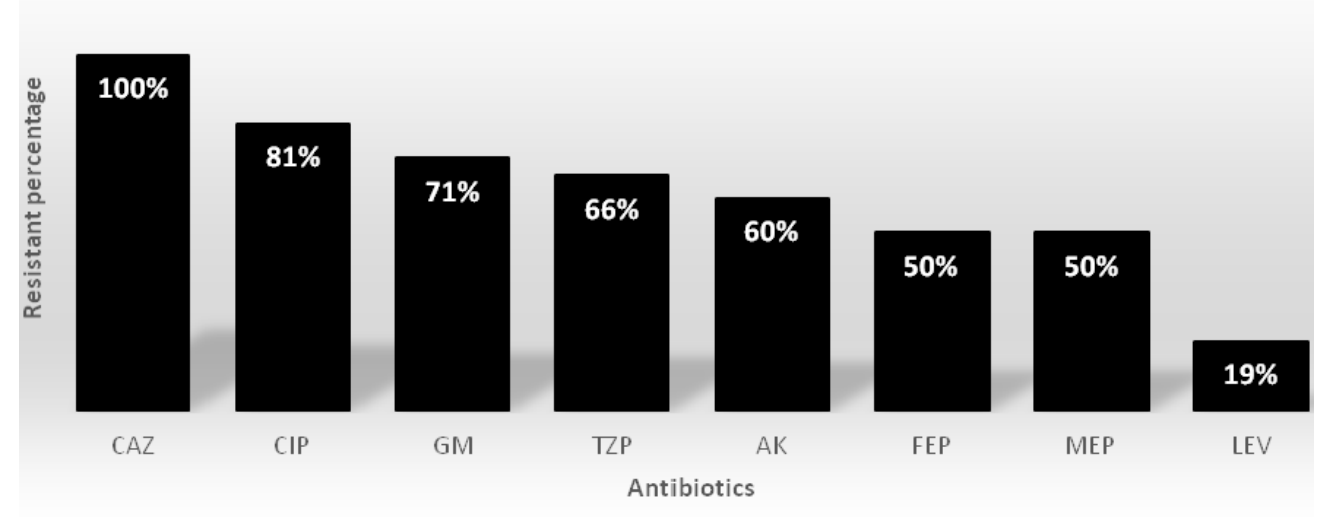

Chart 1. In vitro susceptibilities for 62 clinical isolates of pandrug-resistant $A$. baumannii (PDRAB) determined by disk diffusion [CAZ (Ceftazidime), CIP (Ciprofloxacin), GM (Gentamicin), TZP (Piperacillin/tazobactam), AK (Amikacin), FEP (Cefepime), MEP (Meropenem), LEV (Levofloxacin)]

Table 2. MIC value of colistin in the isolated A. baumannii PDR strains of Shahid Motahari Burn Hospital

\begin{tabular}{lccccc}
\hline & Killing activity & & & Total \\
\hline MIC value & 0.5 & 2 & 4 & 6 & - \\
No. of strains & 0 & 0 & 7 & 2 & $9(14.5 \%)$ \\
\hline
\end{tabular}


from Iran (15) showed that out of the $60 \mathrm{~A}$. baumannii isolates, $57(95 \%)$ were MDR and $11.6 \%$ of the total strains were colistin-resistant (compared to $14.5 \%$ colistin-resistant in the current study). Moreover, results of the disk diffusion method showed that $A$. baumannii isolates had the highest resistance rate against gentamicin and ciprofloxacin (100\% resistance) and the lowest resistance rate against imipenem ( $83 \%$ resistance). In our study, the resistance rates against gentamicin and ciprofloxacin were $70 \%$ and $80 \%$, respectively.

In this study, out of the 115 clinical $A$. baumannii strains isolated from burnt children in Tehran, Iran, 36 (58 \%) were recognized as MDR, $17(27.5 \%)$ as XDR and nine isolates $(14.5 \%)$ as PDR. There are a few published pieces of literature reporting multidrug resistance among clinical isolates of A.baumannii in Iran and, to our knowledge, our study is the first report of such isolates among burnt children.

A more detailed study of molecular pathogenesis and mechanisms of antibiotics resistance among XDR and PDR strains is critical to overcoming challenges facing the treatment of such isolates. Furthermore, the discovery and development of new therapies and precise administration of the existing antimicrobial regimens based on antibiotics susceptibility tests will certainly reduce the high prevalence of resistant strains $(16,17)$.

\section{Conclusion}

The current data indicated a high rate of resistance among Acinetobacter baumannii isolates from burnt children. Since remedial choices are limited for multidrug-resistant A. baumannii infections, the finding and expansion of novel remedies, well-controlled administration of the antimicrobial diets, as well as a greater emphasis on strategies of preventing healthcare-associated transmission of multidrugresistant $A$. baumannii infections are urgent.

\section{Acknowledgments}

The authors wish to extend their gratitude to Iran University of Medical Science for financial support and sponsorship.

\section{Conflict of Interests}

The authors declare that they have no competing interests. definitions for acquired resistance. Clin Microbiol Infect. 2012;18(3):268-81.

7. Peleg AY, Seifert H, Paterson DL. Acinetobacter baumannii: emergence of a successful pathogen. Clin Microbiol Rev. 2008;21(3):538-82

8. Peleg AY, de Breij A, Adams MD, Cerqueira GM, Mocali S, Galardini $\mathrm{M}$, et al. The success of Acinetobacter species; genetic, metabolic and virulence attributes. PloS One. 2012;7(10):e46984.

9. Kallstrom G. Are quantitative bacterial wound cultures useful? J Clin Microbiol. 2014;52(8):2753-6.

10. Bauer A, Kirby W, Sherris JC, Turck M. Antibiotic susceptibility testing by a standardized single disk method. Am J Clin Pathol. 1966;45(4 ts):493-6.

11. Mirshekar M, Shahcheraghi F, Azizi O, Solgi H, Badmasti F. Diversity of class 1 integrons, and disruption of carO and dacD by insertion sequences among Acinetobacter baumannii isolates in Tehran, Iran. Microb Drug Resist. 2018;24(4):359-66.

12. Hojabri Z, Pajand O, Bonura C, Aleo A, Giammanco A, Mammina C. Molecular epidemiology of Acinetobacter baumannii in Iran: endemic and epidemic spread of multiresistant isolates. J Antimicrob Chemother. 2014;69(9):2383-7.

13. Maslow JN, Glaze T, Adams P, Lataillade M. Concurrent outbreak of multidrug-resistant and susceptible subclones of Acinetobacter baumannii affecting different wards of a single hospital. Infect Control Hosp Epidemiol. 2005;26(1):69-75.

14. Bialvaei AZ, Kouhsari E, Salehi-Abargouei A, Amirmozafari N, Ramazanzadeh R, Ghadimi-Daresajini A, et al. Epidemiology of multidrug-resistant Acinetobacter baumannii strains in Iran: a systematic review and meta-analysis. J Chemother. 2017;29(6):327-37.

15. Vakili B, Fazeli H, Shoaei P, Yaran M, Ataei B, Khorvash F, et al. Detection of colistin sensitivity in clinical isolates of Acinetobacter baumannii in Iran. J Res Med Sci. 2014;19(Suppl 1):S67.

16. Eliopoulos GM, Maragakis LL, Perl TM. Acinetobacter baumannii: epidemiology, antimicrobial resistance, and treatment options. Clin Infect Dis. 2008;46(8):1254-63.

17. McArthur AG, Waglechner N, Nizam F, Yan A, Azad MA, Baylay $\mathrm{AJ}$, et al. The comprehensive antibiotic resistance database. Antimicrob Agents Chemother. 2013;57(7):3348-57.

\section{References}

1. Kouchak F, Askarian M. Nosocomial infections: the definition criteria. Iran J Basic Med Sci. 2012;37(2):72.

2. Sobouti B, Khosravi N, Daneshvar A, Fallah S, Moradi M, Ghavami Y. Prevalence of beta lactamase producing species of pseudomonas and acinetobacter in pediatric burn patients. Ann Burns Fire Disasters. 2015;28(3):171-77.

3. Hakyemez IN, Kucukbayrak A, Tas T, Yikilgan AB, Akkaya A, Yasayacak A, et al. Nosocomial Acinetobacter baumannii infections and changing antibiotic resistance. Pak J Med Sci. 2013;29(5):1245.

4. Enani MA. Antimicrobial resistance: insights from the declaration of world alliance against antibiotic resistance. Saudi Med J. 2015;36(1):11.

5. Kanungo R. Antimicrobial resistance: Action by laboratories today for a cure tomorrow. Indian J Med Microbi. 2011;29(3):205.

6. Magiorakos AP, Srinivasan A, Carey R, Carmeli Y, Falagas M, Giske

$\mathrm{C}$, et al. Multidrug-resistant, extensively drug-resistant and pandrugresistant bacteria: an international expert proposal for interim standard 